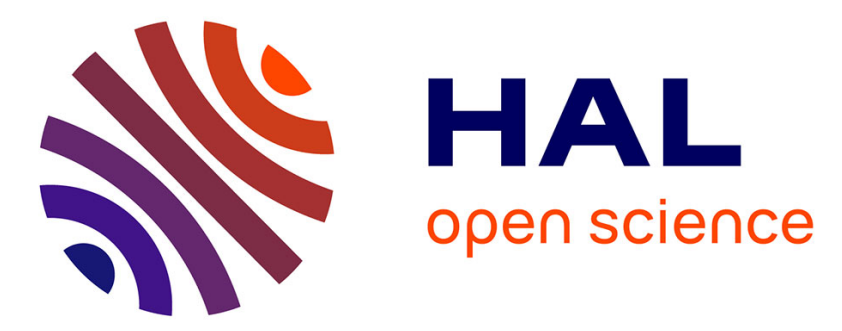

\title{
L'espace et le territoire dans l'agenda de recherche en stratégie
}

Jacques Lauriol, Véronique Perret, Franck Tannery

\section{To cite this version:}

Jacques Lauriol, Véronique Perret, Franck Tannery. L'espace et le territoire dans l'agenda de recherche en stratégie. Revue Française de Gestion, 2008, 34 (184), pp.181-198. 10.3166/rfg.184.181-198 . halshs-00536285

\section{HAL Id: halshs-00536285 \\ https://shs.hal.science/halshs-00536285}

Submitted on 15 Nov 2010

HAL is a multi-disciplinary open access archive for the deposit and dissemination of scientific research documents, whether they are published or not. The documents may come from teaching and research institutions in France or abroad, or from public or private research centers.
L'archive ouverte pluridisciplinaire HAL, est destinée au dépôt et à la diffusion de documents scientifiques de niveau recherche, publiés ou non, émanant des établissements d'enseignement et de recherche français ou étrangers, des laboratoires publics ou privés. 


\title{
L'espace et le territoire dans l'agenda de recherche en stratégie
}

\author{
Jacques Lauriol, Véronique Perret, Franck Tannery
}

Paru dans, Revue Française de Gestion, Vol 34, n¹84, pp 181-198, 2008.

La vitesse des échanges et des relations ouverte par les technologies de l'information et de la communication dissoud certaines contraintes temporelles par la connexion parallèle et simultanée d'une multitude d'acteurs. Certains blocages inhérents aux distances sont aussi modifiés puisqu'il devient possible d'échanger, de communiquer, et de concevoir ensemble dans des lieux différents. Pourtant, de manière paradoxale, ces nouvelles potentialités n'ont fait que renforcer la centralité de l'espace comme facteur structurant de la polarisation des activités autour de certains territoires, comme la Silicon Valley figure emblématique de ce nouveau monde des technologies de la communication. Même l'avénement du virtuel, qui permet des dédoublements temporels avec la vie quasi autonome d'avatars, impose une modélisation de l'espace. L'homme artificiel ou virtuel ne saurait exister sans un retour réflexif sur les espaces de l'homme.

L'espace et le territoire se retrouvent ainsi aujourd'hui au coeur de renouveaux théoriques en sciences humaines et sociales, comme pour les grandes oeuvres théoriques passées en sociologie ou en économie si nous pensons à Weber, Marshall ou Ricardo. Ce renouveau s'est imposé pour affronter des questions aussi vastes et multiples que la mondialisation de l'économie, l'hypercroissance ou l'appauvrissement de certaines régions, le développement durable d'activités et de territoires, l'émergence de nouvelles entreprises globales sur la scène internationale issues de «nouveaux» pays (Corée, Mexique, Inde par exemple), la répartition des activités d'innovation et de recherche et développement, les effets des transformations techniques sur l'organisation spatiale du système économique et social, -ou encore l'animation d'acteurs territoriaux aux intérêts disparates, etc... L'article de J-B Zimmermann expose ce renouveau dans ce dossier par une présentation très claire du point de vue actuel de l'économie sur l'espace et le territoire.

La stratégie et la gestion ne sauraient rester en marge de cet effort d'interrogation théorique et de proposition conceptuelle. Cela justifie de mener ici un effort de repérages des principaux enjeux reliants stratégies, espaces et territoires. Ce travail se fera en distinguant deux aspects. Dans un premier temps nous aborderons la dimension spatiale des stratégies des firmes et des organisations qui nécessite d'interroger conjointement la localisation des activités et le management stratégique des distances. 
Dans un second temps nous reviendrons sur le territoire comme stratégie d'organisation de l'espace. II s'agit notamment d'interroger les formes d'organisation spatiales, les modes de coordination et enfin les modalités de gouvernance d'acteurs disparates. Comme l'a montré dernièrement C-A.Michalet (2007), lors de sa dernière contribution à la Revue Française de Gestion sur la «Dynamique des formes de délocalisations et gouvernance des firmes et des Etats», ces deux questions sont foncièrement liées. Leur articulation justifie la proposition d'un agenda de recherche à la fin de ce propos d'étape.

\section{La dimension spatiale des stratégies}

La question de la distribution spatiale des activités au sein des firmes et des industries a été abondamment traitée par la littérature, tant d'un point de vue théorique que d'un point de vue empirique. Avec Perrat et Zimmermann (2003), on peut considérer que les travaux visent en dernier ressort à appréhender les dynamiques de formation et d'offre de ressources co-construites entre organisations, entreprises et territoires. Ces dynamiques contribuent à la formation fructueuse et durable d'avantages compétitifs mutuels. Outre la question spécifique des territoires sur laquelle porte la partie suivante, il s'agit d'interroger dans un premier temps les stratégies des firmes et des organisations pour comprendre les choix qu'elles opèrent quant à la localisation de leurs activités selon un continuum articulé autour de stratégies « nomades » et de stratégies « d'ancrage » durable dans un territoire. Ces choix dépendent du management stratégique de l'espace et des distances mis en oeuvre par les firmes et les organisations car celui-ci conditionne la localisation de la formation des ressources spécifiques et des capacités dynamiques.

\section{Localisation des activités ou l'espace comme variable du design organisationnel}

Le fait qu'un numéro spécial de Advances in Strategic Management (2003) soit consacré à «Geography and Strategy» constitue un signe manifeste de l'importance prise par la question spatiale en stratégie. Dans l'introduction à ce numéro spécial Sorenson et Baum (2003) proposent de répartir les travaux en deux catégories.

II y a tout d'abord ceux qui abordent la stratégie de localisation à partir de la question de la « place » en considérant que les performances supérieures obtenues par la localisation dans une région du monde résultent des caractéristiques et attributs particuliers du territoire considéré. N'étant pas mobiles, ces attributs ne s'avèrent disponibles que dans un nombre limité de localisations. Cela confère à ces 
localisations un avantage et favorise une dynamique d'agglomération autour. Tel est le cas des ressources scientifiques disponibles en matière de biotechnologies (Stuart et al, 2003). II en est de même pour les cas emblématiques de la Silicon Valley et de la route 128, dont les spécificités culturelles centrées sur la coopération, l'existence d'un contexte institutionnel très particulier et la concentration importante de ressources variées -technologies, qualification des personnels, industrielles...- expliquent la forte et continuelle attractivité de ces territoires (Hall et al, 2001). Cette analyse en terme de place peut se résumer par l'image ou le vieux principe d'action selon lequel «au bord des rivières seront les moulins» (Lavenir et al., 2001 : 31).

II y a ensuite les travaux qui abordent la localisation à partir de la question de "l'espace » en s'intéressant à la distribution spatiale des firmes à l'intérieur d'une industrie ainsi qu'à la répartition des actifs et des ressources au sein des firmes elles-mêmes. Ces deux questions ont des conséquences sur l'organisation des relations entre industries et sur les déterminants de leur spatialisation (Elango, 2004). Ce mouvement de "pooling " amène des firmes aux activités différenciées à partager où à s'associer autour de ressources communes en raison de l'existence de «Knowledge Spillovers» fondés sur des réseaux sociaux inter et intra firmes. Ces «spillovers» sont à l'origine de dynamiques de « contagion spatiale » tant à l'intérieur des firmes elles-mêmes (qui détermine la vitesse de diffusion interne d'innovations dans des firmes aux localisations multiples), qu'entre firmes exerçant des activités différentes mais également parties prenantes de ces réseaux sociaux (Bala et al, 1998 ; Sorenson et al, 2003). En d'autres termes, l'interrogation porte sur la position des acteurs dans des systèmes de liens sociaux situés spatialement, ainsi que dans des systèmes plus globaux non directement liés à un ancrage spatial mais relatifs à l'organisation interne de la firme. La combinaison de ces facteurs forment ces « attracteurs » de la localisation spatiale des firmes. Ces réseaux peuvent être à l'origine de rentes relationnelles (Dyer et al, 1998) entre membres, pour autant que certaines conditions soit respectées (spécificité des actifs engagés, complémentarité des ressources mobilisées, gouvernance appropriée...).

Ces deux angles de questionnement de la localisation (place / espace) présentent le mérite de se focaliser sur des facteurs explicatifs. Force est en effet de constater que l'activisme des entreprises provoque une obsolescence rapide des cadres analytiques, ou du moins leur insuffisance manifeste pour couvrir le champ des enjeux de localisation et la géographie de la mondialisation (Carroué, 2002). L'article de A.Colovic et U.Mayhrofer dans ce dossier illustre bien cette situation dans le cas du secteur de la construction automobile mondiale. Cela justifie les efforts réguliers de la CNUCED pour suivre les évolutions des pratiques, notamment la rapide émergence de groupes mondiaux issus de nouveaux pays (Mexique, Bresil, Inde, Chine, Corée, etc.) (The Economist 2008). II faut aussi tenir compte des 
changements permanents dans les périmètres des groupes, notamment via les opérations de fusion et d'acquisition.

Devant cette difficulté méthodologique la principale voie choisie en stratégie reste l'étude de cas (Pellenbarg et al., 2008) pour analyser tel ou tel phénomène : les réseaux locaux d'entreprises dans une région, l'organisation spatiale de la production d'un groupe, la répartition spatiale au sein d'un secteur, l'imbrication de réseaux locaux et globaux, etc. Cette approche permet de contourner l'écueil de certains travaux qui restent cantonés à l'analyse d'une dotation de facteurs économiques (fiscalité, main d'oeuvre, infrastructures, foncier, etc.). L'enjeu est effectivement de parvenir à prendre en compte l'effet structurant des processus de décision sur les options de localisation (Sergot, 2007). Ces processus de décision, et plus généralement le style de management, déterminent la répartition spatiale des fonctions d'innovation et de conception au sein des groupes (Tannery et al, 2007). Pour le management stratégique, cela revient à cerner les différentes formes possibles d'organisation et de stratégie des activités en fonction du niveau d'avantages spécifiques tant de l'entreprise que de ceux qui résident dans la localisation elle-même (Rugman, 2005). En cela la géographie et l'espace deviennent des variables centrales pour le design organisationnel des groupes (Westney, 2003). Le marketing ne s'y est pas trompé en développant tout un courant de recherche sur le géomarketing. Ce courant pourrait, à certains égards, être croisé avec les recherches sur les stratégies multi marchés (Mcgrath et al., 1998). Quoiqu'il en soit, cette variable spatiale devient d'autant plus centrale que les décisions prises nécessiteront simultanément de développer des outils de gestion pour assurer le management des distances comme nous allons l'envisager maintenant.

\section{Le management stratégique de l'espace et des distances}

Globalement les analyses précédentes sur la localisation des activités partagent une préoccupation commune : celle des ressources et des actifs qui peuvent être soit localisés dans un territoire doté d'attributs intrinsèques qui forment son attractivité, soit au contraire, distribués spatialement entre firmes, territoires et à l'intérieur même des firmes. La question qui se pose ici est celle de la spécificité spatiale de ces ressources et de leur dynamique de construction.

Le caractère générique d'une ressource et d'un actif territorial réside dans son caractère de transférabilité selon des mécanismes de marché, d'un espace ou d'un territoire à un autre (Colletis et al, 2004, Pecqueur, 2005). Les ressources et actifs spécifiques se caractérisent eux par leur caractère non transférable du fait des coûts d'irréversibilité que toute tentative de réaffectation à un autre espace pourrait générer. Ces spécificités proviennent de la singularité de leur construction, des réseaux sociaux 
à l'origine de la production de connaissances, des qualifications des personnels, des coopérations entre acteurs territoriaux, etc. La seule manière d'accéder à ces ressources et actifs spécifiques, qui expriment le caractère différencié d'un territoire à l'autre, passe par une forme d'ancrage territorial. Cet ancrage permettra d'articuler la logique de développement de l'entreprise, la logique industrielle qui caractérise un secteur, et les ressources ou actifs spécifiques disponibles localement pour résoudre des problèmes productifs.

Le caractère cumulatif de la production et de l'exploitation de ces ressources et actifs spécifiques autour de dynamiques collectives d'apprentissage et de processus de co-production, amène les acteurs à investir ensemble sur des ressources similaires en contribuant ainsi à une certaine spécialisation spatiale ou territoriale (Perrat et al, 2003). Cette spécialisation peut s'exprimer par des trajectoires technologiques locales spécifiques. En même temps, ces acteurs doivent également coordonner un ensemble de ressources complémentaires, non similaires parce que liées aux compétences spécifiques qu'ils détiennent individuellement cette fois-ci (Boulba-Olga, 2006). L'ensemble de ces questions nécessiteront de faire des choix en matière de management de l'espace en interrogeant où et comment seront articulées telle ou telle activité, telle ou telle ressource. Les principales analyses portent notamment sur les activités d'innovation, lors de l'internationalisation des entreprises, en interrogeant celles qui sont menées dans le pays d'origine et celles qui sont menées dans le pays d'accueil. La qualité de l'encastrement local, l'histoire des unités, l'ancienneté de l'implantation, le type d'innovation ou encore la capacité d'exploration de l'unité locale sont certaines des questions à prendre en compte (Frost, 2001).

Plus généralement, se pose le problème de la coordination de ces ressources et de l'organisation spatiale nécessaire à leur structuration, à leur mobilisation et à leur valorisation au sein de l'entreprise. Ce questionnement a permis récemment un certain renouveau des questions sur le management des firmes multinationales (Rugman et al, 2001). II s'agit d'abandonner l'attention dominante sur les types de stratégies internationales pour revenir plus précisément sur le rôle des unités au sein des entreprises. Ce courant, qui va de pair avec l'intérêt croissant porté au management intermédiaire ou aux pratiques stratégiques, a notamment mis en lumière que les unités pouvaient développer de fortes capacités d'initiatives, et être source d'innovation de management (Birkinshaw, 1997). Ces unités sont en définitive des acteurs locaux qui contribuent à la structuration du jeu global de la multinationale (normes, projets d'innovation, pratiques de gestion) comme le démontrent avec une rare finesse Kristensen et Zeitlin (2005) à travers l'histoire d'une multinationale fruit de l'intégration de multiples entreprises et de multiples compétences locales. De son côté Rugman (2005) propose de distinguer plusieurs cas de figure en fonction de la répartition des processus de décision ou de pouvoir dans 
l'entreprise (siège, centre régional, filiale nationale) et des caractéristiques des produits (global, région, local). Chaque configuration prêtera en partie à conséquence sur la circulation des connaissances et des ressources entre les unités et au sein de l'entreprise.

Cette question du transfert des connaissances constitue probablement un enjeu plus général pour le management de l'espace et des distances. Les très nombreuses recherches sur le management des connaissances, de l'innovation ou de l'apprentissage contournent trop souvent cette question. Elles font ressortir les caractéristiques des phénomènes (exploration vs exploitation par exemple) en questionnant en définitive assez peu où et comment se déroulent les processus. Pourtant les apprentissages sont situés (Tyre et Von Hippel, 1997) et les environnements locaux dans lesquels ils ont lieu importent particulièrement. Cette question prête à conséquences sur la manière de concevoir le rôle de l'expertise et des relations entre les acteurs au sein des organisations ou entre les organisations. Dans ce dossier, le texte de S. Adam-Ledunois et S. Renault sur les Parcs Industriels Fournisseurs analysent bien ce phénomène. Ces parcs s'avèrent un des dispositifs de gestion de l'espace pour rapprocher des acteurs, leur permettre d'échanger et de développer mutuellement des connaissances tout en leur assurant de garder une autonomie relative. Ces parcs contribuent aussi à structurer le territoire économique d'implantation des entreprises en organisant une certaine répartition spatiale des connaissances et des ressources en leur sein.

\section{Le territoire comme modalité d'organisation de l'espace.}

La perspective de la ressource, qui considère le territoire comme une sorte de patrimoine de ressources spécifiques (François et alii, 2006), permet de poser un premier constat. Le territoire est une entité qui opère comme une organisation spatiale complexe, économique, sociale et politique et comme mode d'organisation des relations entre un ensemble d'agents situés localement. II n'est donc pas « réduit à sa seule dimension spatiale... un espace neutre, un réceptacle de technologies... mais il doit être considéré comme un vecteur indissociable de leur émergence et de leur développement » (Sierra 1997: 10). II ne s'agit, en aucun cas, de s'inscrire dans une sorte de "préjugé localiste » qui définirait le territoire comme « un donné» composé d'acteurs dotés de ressources qu'ils mobilisent dans un cadre territorial. Tout au contraire, il s'agit de considérer une organisation territoriale qui «maille » ou «encastre différents acteurs porteurs de rationalités diverses et limitées, différents niveaux d'intervention (le local, le national, le global...), différentes institutions et organisations dont les logiques 
d'action peuvent parfois se révéler incompatibles... autour de projets et d'intérêts communs » (Lauriol, 2006: 367).

Le territoire se conçoit comme une forme d'organisation de l'action collective spatialisée et territorialisée. C'est à ce titre que le territoire devient une question pour la stratégie et amène à envisager trois problèmes stratégiques :

- Quelles sont les formes d'organisation de cette action collective nécessaires à l'obtention d'une performance satisfaisante?

- Quelles sont les modes de coordination les plus appropriés au développement de coopérations fructueuses pour l'ensemble des acteurs concernés?

- Enfin, quelle gouvernance mettre en phase pour réguler les interactions qui se déroulent dans ces organisations territoriales?

\section{Les formes d'organisation de l'action collective territorialisée.}

Depuis le début des années 90, la question les formes d'organisation de l'action collective territorialisée a fait l'objet de nombreuses analyses. Outre la grande diversité des formes envisagées et proposées, on ne peut que constater aussi une forte polysémie.

Cette diversité de formes a pour origine le concept de district industriel, proposé par A. Marshall (1890 1961), qui a lui-même suscité d'importants travaux (Benko et alii, 1997). Un district industriel « est une entité socio-territoriale caractérisée par la présence active d'une communauté de personnes et d'une population d'entreprises, dans un espace géographique donné » (Beccatini, 1982: 36 in Benko et alii, 1997). On trouve, dans la proposition de Marshall, l'essentiel des concepts mobilisés aujourd'hui par l'économie spatiale et la nouvelle géographie économique (le concept d'externalité, celui d'agglomération, d'atmosphère industrielle, de proximité, de système d'interactions, de culture...). C'est sans doute la richesse de cette conceptualisation qui explique l'intérêt qu'elle suscite aujourd'hui encore.

En tant qu'organisation industrielle territorialisée, un district industriel a été analysé et requalifié comme un réseau porteur d'externalités innovantes (les réseaux d'innovation Cohendet et alii, 1998), comme étant à l'origine d'une dynamique endogène de développement (les milieux innovateurs Maillat, 1995 ; les Learning Region, Florida, 1995), comme communauté d'apprentissage (Loasby, 2002), ou encore comme système productif local (Datar, 2004) ce dernier privilégiant l'agglomération d'acteurs autour d'une filière. Les technopôles et les récents pôles de compétitivité, s'inscrivent eux dans une logique 
qui est celle de la théorie de la polarisation proposée par F. Perroux (1961), qui mobilise elle aussi le concept d'externalité d'origine Marshalienne. La notion de pôle de compétitivité présente, selon la définition qui en a été proposée par C. Blanc (2004), la particularité « d'agglomérer » en quelque sorte, la quasi-totalité des dimensions précédentes: un pôle de compétitivité s'appuie sur une dynamique endogène fondée sur l'innovation technologique, sur une proximité géographique entre acteurs ainsi que sur l'existence d'un cluster industriel (Blanc, 2004: 26).

C'est cette dernière appellation de cluster semble s'imposer aujourd'hui, malgré l'absence de consensus sur une définition stabilisée. Elle désigne un groupe de firmes interconnectées appartenant à une même industrie ou à des industries reliées, géographiquement localisées et qui bénéficient de ce fait d'une dynamique d'agglomération et d'une capacité d'innovation supérieure par l'exploitation d'un savoir commun (Bell, 2005 ; Porter, 2000).

Cette proposition « néo-Marshallienne » tient plus de la « métaphore » que du concept du fait de son caractère élevé de généralités, de l'imprécision et de la variabilité qui caractérisent ses éléments constitutifs (l'échelle d'un cluster : local, régional, national ?, la question de ses frontières, la notion de compétitivité, les « drivers » d'un cluster... (Martin et al, 2003 ; Brown et alii, 2007). Après analyse, cette notion de cluster parvient à combiner et à se référer à trois théories principales (Gordon et al, 2000).

- Les économies d'agglomération qui se focalisent sur les externalités induites par la concentration géographique.

- Le modèle du complexe industriel, central dans l'approche en termes d'économie régionale, dans laquelle la concentration géographique résulte de la recherche de minimisation des coûts de transaction.

- La théorie des réseaux sociaux, qui analyse le cluster comme un ensemble de relations interpersonnelles organisées en réseaux socialement encastrés.

Au-delà de ces idéaux-types, le caractère "chaotique » et excessivement marketé ("cluster as a brand », Martin et al, op cit) de la proposition de Porter $(1990,1998)$ nécessiterait d'interroger plus avant sa pertinence empirique (The Economist 2007) voire même sa pertinence théorique (Davies et al, 2000). Quoiqu'il en soit il y a là une institutionalisation, trace d'un indéniable succès qui permet de considérer également le cluster en tant que « mythe rationnel » c'est-à-dire (une) « conception limitée $d u$ monde... qui favorise une dynamique de l'action collective... et... une nouvelle perception des relations... même s'il est...vague et ambigu. » (Hatchuel, 2000: 38). C'est en ce sens qu'il présente un grand intérêt du fait même des interrogations et investigations qu'il suscite. Nous en avons une bonne illustration dans ce dossier avec l'article de Suire et Vicente qui l'applique à l'analyse des réseaux 
d'entreprises innovantes. Plus généralement, au delà des seules formes d'organisation de l'action territoriale, il invite à s'intéresser aux modalités de coordination. Cette question, tout aussi essentielle, est analysée dans la prochaine section.

\section{La coordination de l'organisation territoriale}

Les modalités de la coordination territoriale présentent une grande diversité, à l'instar des formes d'organisations territoriales. Sur le plan stratégique il s'agit de combiner à la fois des actions plutôt focalisées, par exemple autour d'une firme pivot centrale pour tel ou tel projet, et des actions distribuées mobilisant une pluralité d'acteurs. L'ensemble repose sur des dynamiques d'apprentissage et la formation d'une sorte de connaissance et d'un langage communs entre agents, l'ensemble contribuant à l'émergence de réseaux territorialisés (Callon, 1999). Dans ce dossier, l'éclairage apporté par le texte de Nathalie Raulet Croset sur la dimension territoriale des situations de gestion montre l'importance de la constitution de ces réseaux pour traiter de problèmes spatialement situés.

II apparaît ainsi que les dimensions cognitives doivent être considérées comme centrales dans les problématiques de coordination, qu'il s'agisse des processus d'apprentissage qui permettent de former une connaissance commune au sein d'un territoire ou des conventions qui, en tant que dispositifs cognitifs collectifs (Orléan, 1994), constituent des systèmes "d'interdépendances hors marché » (Storper, 1995: 115). L'unité analytique de base utilisée pour rendre compte de ces dynamiques cognitives est souvent celle de la communauté (Cohendet et al., 1998), dans laquelle la confiance entre membres exerce une influence essentielle sur la cohésion interne de cette communauté et sur la capacité de ses membres à collaborer sur le plan local ou pour développer des stratégies multi territoriales (Adler et al, 2005). La production de cette confiance s'appuie soit sur des dispositifs attachés à des personnes insérées dans des réseaux de relations, soit sur des «dispositifs de promesse » (Karpik, 1996) relatifs à la qualité de la personne elle-même (sa réputation) ainsi qu'à des dispositifs normatifs coproduits entre partenaires contractuels, au sein de "systèmes industriels locaux » par exemple. Ces questions inspirent en particulier les recherches sur les programmes d'accompagnement de l'entrepreneuriat ou sur les entrepreneurs et leurs réseaux (Hite, 2003). La relation de confiance qui se construit dans l'interaction au travers de ces dispositifs à vocation normative, mêle dimensions culturelles, économiques, sociales, politiques et morales façonnées par des acteurs qui partagent une expérience commune. Cette expérience de nature communautaire s'avère encastrée dans des « contextes locaux d'interactions » (Dupuy et al, 2004: 66 et 79). On peut alors considérer que si ces «organisations locales s'appuient sur des relations de type confiance 
domestique (ou communautaire), alors elles sont fortement ancrées dans l'espace. Dans ce cas, confiance rime avec proximité. » (Dupuy et al, 2004: 81).

Ce concept de proximité, et ses différentes déclinaisons, semble s'imposer aujourd'hui comme un élément majeur de l'analyse de ces problématiques de coordination dans l'organisation territoriale. II permet d'interroger la nature et la configuration des interrelations qui peuvent relier des activités situées dans un territoire mais aussi des acteurs au sein d'une même organisation dont certaines entités peuvent être localisées dans un espace géographique particulier. Ces acteurs, pour reprendre la proposition de Rallet (1999), sont ainsi « là » (dans un territoire) et «ailleurs», c'est-à-dire inscrits dans des réseaux « extra territoriaux ». Dans le premier cas, on considère une proximité géographique qui « traduit la distance kilométrique entre deux entités, pondérée par le coût temporel et monétaire de son franchissement» (Torre et al, 2005: 49). Dans le deuxième cas, on s'intéresse à une proximité organisée et relationnelle qui désigne « la capacité d'une organisation à faire interagir ses membres » (ibid: 49). Cette proximité organisationnelle s'appuie sur des dimensions cognitives (croyances, représentations...) et se déploie à l'intérieur d'une organisation dont les frontières ne sont pas uniquement fixées par un territoire (une organisation transnationale, un réseau...). Elle mobilise une autre forme de proximité, institutionnelle cette fois ci, qui « exprime l'adhésion d'agents à un espace commun de représentations, de règles d'action et de modèles de pensée » (Kirat et al 2001: 212). Le terme institutionnel désigne à la fois des organisations (entreprises, réseaux...) et des comportements (règles, routines, normes au sens de North, 1990), "l'ensemble de ces formes contribuant à la formation et à la régulation des espaces locaux » (Kirat et al., ibid).

Le caractère « ambigu » (Torre et al, 2005) et polysémique (Sierra, 1997) du terme de proximité participe d'une certaine « inflation » dans la création de nouvelles formes de son expression (Boschma 2005b). II en va de même pour le recours à la question de la confiance. II reste important de travailler à une meilleure définition de ces dimensions analytiques dans une perspective plus dynamique, qui permettrait de comprendre l'émergence, le développement, la disparition ou la substitution entre les différents modes de coordination de l'organisation territoriale. Malgré la variété de ces approches, ces dynamiques de confiance et de proximité s'avèrent aujourd'hui très structurantes pour décrire, comprendre et tenter d'expliquer les modes de coordination à l'œuvre dans ces différentes formes d'organisations territoriales. De nombreux travaux de terrain en témoignent (Gilly et al, 2001 ; Perrat et al, 2003 ; Schamp et alii, 2005). Au-delà des problèmes de coordination qu'ils analysent, ils soulèvent également la question de la gouvernance territoriale et des mécanismes de régulation qu'elle met en œuvre pour contrôler et stabiliser durablement le fonctionnement de ces organisations territoriales, et les aider à faire face aux problèmes inédits auxquelles elles peuvent être confrontées. 


\section{La Gouvernance Territoriale}

L'action territoriale mobilise des acteurs dont les attentes et logiques d'action sont plurielles. Le pilotage de cette action collective nécessite l'activation d'un système de régulation capable de gouverner durablement cette pluralité, en assurant compatibilité, complémentarité et convergence des acteurs autour de projets communs. C'est à ce titre que l'étude des territoires s'avère particulièrement riche d'intérêt pour la recherche en stratégie.

Cette gouvernance territoriale, définie comme un "processus institutionnel - organisationnel de construction d'une mise en compatibilité de différents modes de coordination entre acteurs géographiquement proches » (Gilly et al, 2001) s'appuie principalement sur trois régulations :

- Le mode de coordination des activités et des relations entre auteurs ;

- Les processus de décision stratégique et d'allocation des ressources ;

- enfin, le contrôle de l'activité, par l'exercice du pouvoir et la régulation des conflits qui se manifestent dans la recherche de compromis et d'accords.

L'articulation de ces régulations donne forme à cinq idéaux-types de gouvernance (Le Galès, 2004: 109) :

- Le marché où la régulation s'effectue à partir de la concurrence et des prix, que l'on retrouve en partie dans les districts de la « Troisième Italie » (Aniello, 2001).

- La hiérarchie avec une gouvernance à dominante privée impulsée par la grande firme qui structure l'espace productif local (l'industrie automobile ou pétrochimique en Haute-Normandie, par exemple).

- La gouvernance publique qui procède, avec l'Etat, par la contrainte ou l'incitation exercées via la réglementation et par l'influence des différentes instances publiques locales (agences, collectivités territoriales,...)

- L'association, autre forme de gouvernance privée, qui mobilise des opérateurs professionnels autour d'institutions locales "en charge de l'élaboration, de l'adaptation et de l'exécution des règles collectives » (Ehlinger et alii, 2007: 164). II peut s'agir de syndicats, d'association d'entrepreneurs, de chambres de commerce... qui représentent leurs membres auprès des autorités publiques. 
- Et enfin, la communauté, qui repose sur des solidarités et des identités autonomes articulées autour de relations de confiance (les communautés de pratiques par exemple).

Comme il s'agit d'idéaux-types, du fait de la singularité de chaque territoire et de la variabilité des jeux d'acteurs qui modèlent chaque période de sa construction, on observe un certain mixage de ces modes de gouvernance et de régulation territoriales. Selon les cas, on observera une dominante plus ou moins forte de l'un d'entre eux (Perrat et al, 2003). Tel fut le cas, par exemple, pour le pôle aérospatial de Toulouse dont la gouvernance territorialisée s'avère « une combinaison de régulations de marché... publique... de grandes entreprises et d'un soupçon de communauté et d'association » (Le Galès, 2004: 121). La gouvernance de ce pôle s'est en effet construite à partir d'un « enchevêtrement » de modes de régulation dominants ; publique tout d'abord, par la décision d'implanter à Toulouse un potentiel de recherche et des industriels de ces secteurs; puis privée, par l'introduction de la hiérarchie impulsée par quelques grandes firmes ; puis le marché par la constitution de réseaux de sous-traitants locaux et aujourd'hui, « un soupçon de communauté et d'association », qu'il s'agisse de groupes de recherche ou de syndicats professionnels qui représentent différents corps de métier au sein de cette organisation territoriale (Gilly, 2005).

Au delà de ce seul cas, la gouvernance territoriale se caractérise par le développement d'une capacité de négociation, notamment pour fonder sa légitimité. Elle ne peut éviter une adaptation en permanence des contenus de l'agrément entre partenaires sur les modalités de mise en œuvre des politiques et des stratégies. En d'autres termes, la gouvernance territoriale offre le prétexte de reposer encore et toujours en stratégie la question de l'exercice du pouvoir et de l'autorité (Ehlinger et alii, 2007). Cette gouvernance territoriale ne peut faire l'économie de mobiliser à la fois des dimensions bureaucratiques et démocratiques (O' Mahony et al, 2007) :

- Bureaucratiques par la définition, plus ou moins claire, des positions hiérarchiques et des attributs juridiques des acteurs au sein du territoire ;

- Démocratiques car ce statut hiérarchique est accompagné de règles qui encadrent cette autorité et qui obligent à rendre compte des résultats obtenus à la communauté.

De ce fait, l'institution de la gouvernance territoriale va de pair avec l'institution des possibilités d'exercice d'un pouvoir « compensatoire », ou contre-pouvoir. II s'agit d'une « autorité distribuée » qui s'appuie sur un processus de délibération interne qui permet d'adapter cette gouvernance aux évolutions d'un contexte ou à la représentation que s'en forment les acteurs.

En conclusion, la gouvernance territoriale se doit de répondre à la diversité des acteurs en présence, à leurs attentes, et aux incertitudes radicales de la coordination de leurs activités et contributions 
respectives à la réalisation d'un projet collectif. Elle permet ainsi de réfléchir aux voies et moyens pour combiner, réguler et contrôler ces enchevêtrements, pour saisir toute la complexité du pilotage de l'action stratégique et politique.

\section{Propositions pour un agenda de recherche}

L'analyse de la thématique "Stratégies, Espaces et Territoires » mobilise des catégories qui sont familières aux sciences de gestion en général et à la stratégie en particulier, qu'il s'agisse des formes d'organisation, de la gestion des ressources ou encore des problèmes de coordination et de gouvernance. La richesse intrinsèque et la variété de ces catégories ouvrent ainsi des avenues de recherche intéressantes qui pourraient structurer un agenda porteur de perspectives probablement très fructueuses lorsque l'on souhaite les appliquer conjointement à la question de l'espace et des territoires. Au niveau des formes d'organisation tout d'abord. Au risque de participer à un accroissement de la diversité de l'offre conceptuelle, il nous semble particulièrement intéressant de considérer le concept d'écosystème. Selon lansiti et al. (2004), un écosystème d'affaires est « un système regroupant des participants faiblement couplés (ou interconnectés) qui répondent les uns des autres pour assurer l'efficacité globale de ce système» (:19). Plus précisément, et pour éviter toute confusion entre écosystème et environnement, Guegen et al. (2004) signalent qu'il ne s'agit pas ici de considérer « toutes les relations que peut nouer une entreprise... mais seulement... les relations directes ou non, entre acteurs guidés par la promotion d'une ressource commune » (:18). Ce faisant, on peut s'attacher à l'analyse des interactions qui concernent la mobilisation d'une ressource territorialisée et ainsi, identifier plus finement le contenu de ces interactions et les influences qu'elles pourraient exercer par exemple, sur les dynamiques d'apprentissage ou la formation de "Knowledge Spillovers». En procédant ainsi on pourrait améliorer la compréhension des stratégies d'ancrage des entreprises ainsi que les dynamiques de structuration des territoires.

II en va de même pour les problèmes de coordination. L'approche par les dynamiques de proximité s'attache pour l'essentiel au repérage des dispositifs de médiation qui soutiennent des interactions entre acteurs membres de réseaux sociaux territoriaux et " extra-territoriaux », et entre activités localisées dans un même espace. D'un point de vue analytique, elle s'intéresse principalement à la mesure de distances entre acteurs (voir par exemple Zimmermann, 2001) plus qu'aux contenus de ces interactions, c'est-à-dire aux contenus des interdépendances qu'elles contribuent à créer. Dans ces conditions, il pourrait être intéressant de développer une grille d'analyse pour croiser le contenu des 
interactions à l'œuvre et l'étude du degré de couplage entre acteurs. Une telle grille permettrait d'évaluer la nature des interdépendances ainsi que leurs effets sur les modes de coordination. L'objectifs serait de parvenir à comprendre la construction de fonctionnalités économiques et sociales (Lauriol, 2007a et 2007b). Cependant, en accord avec les propositions de R.A. Boschma (2005 a), on ne peut que préconiser le développement d'approches multidisciplinaires pour qualifier plus précisément la nature des processus d'apprentissage à l'œuvre, les contenus de ces apprentissages ou encore le jeu des normes et routines et la mesure de leurs influences sur ces dynamiques de coordination

Enfin, compte tenu des analyses précédentes, il nous parait urgent de mettre la recherche au service de la question de la gouvernance de l'espace par les acteurs et de la gouvernance territoriale. Au-delà des formes canoniques du traitement de cette question (les conflits d'intérêts, par exemple), il semble important de s'intéresser de manière plus approfondie à la question du pouvoir, des modalités de son exercice au sein des organisations multi localisées ou des organisations territoriales. II s'agirait notamment de prêter plus d'attention aux différents cognitifs et de conception pour appréhender des processus de délibération stratégique plus ouverts. On pourrait ainsi « s'assurer que l'instance de gouvernance soit à la fois acceptée, reconnue, légitimée par les différents acteurs internes... afin que ceux-ci lui délèguent une partie de leurs pouvoirs de décision, sans pour autant entraver leur autonomie... et... identifier... leur degré d'influence ou de dépendance... et prendre... en compte les jeux de pouvoir » (Ehlinger et alii, 2007: 166-167).

Ce retour sur le pouvoir implique probablement un travail de conceptualisation accrue sur le concept luimême. Ceci permettrait de dépasser l'utilisation standard qui en est généralement faite en matière de management des organisations, à savoir un moyen d'imposition de contraintes locales, organisationnelles, sociétales... Deux voies de dépassement peuvent être envisagées. La première consiste, dans une certaine tradition critique (Avelsson \& Willmott, 2003) à intégrer pleinement la dimension spatiale dans l'analyse des pratiques de pouvoir des organisations et de leur management. Une conception de l'espace telle qu'elle a été présentée dans l'introduction de ce dossier, c'est à dire un espace conçu comme un instrument de pouvoir et une construction sociale, idéologique et politique, invite à un travail de déconstruction. L'espace conçu comme «un artifice» (Lussault, 2007: 18) invite à être dénaturaliser. Certains travaux se sont déjà engagés dans cette voie en analysant par exemple la dimension spatiale de la réthorique managériale sur la globalisation (Banerjee \& Linstead, 2001). Fleming et Spicer (2004) et par la suite Spicer (2006) montrent bien comment la mobilisation d'une échelle spatiale dans le discours stratégique est un acte politique qui établit des limites et des frontières, impose une identité, exclut et inclut certaines logiques. La dénaturalisation des discours et des actes 
spatiaux du management stratégique est une approche intéressante pour engager un travail analytique sur le pouvoir des/dans les organisations. Une seconde voie de dépassement, dont il faudrait envisager plus longuement sa complémentarité avec la première, est de considérer le pouvoir comme un « objet polymorphe et multidimensionnel» qui se structure à partir de combinaisons, "d'interactions et d'interdépendances faisant intervenir différents niveaux, différents champs et différents objets... » pour le penser de manière novatrice par «l'utilisation de concepts adjacents... tels que la résistance et/ou l'hégémonie » (Courpasson et al, 2008).

Retenir cette perspective revient à envisager une ouverture assez novatrice en la matière : celle d'une gouvernance qui se donne pour projet l'exercice de la démocratie. L'investigation pourrait porter ici sur les processus de conception et de construction du mode de gouvernance lui-même, et des configurations d'exercice du pouvoir qui accompagnent cette gouvernance (exercice de l'autorité formelle, processus de contrôle de cette autorité, capacité de contestation ou de « résistance » face à ce qui peut être vécu comme « hégémonique », capacité à délibérer et à « contraindre l'autorité »...). L'analyse de cas de communautés virtuelles, de communautés dites Open Source (O'Mahony et al, 2007) ou encore de communautés collaboratives (Adler et al, 2006) donne quelques indications intéressantes sur la manière dont ces organisations développent une conception partagée de l'exercice de l'autorité à travers l'espace tout en limitant son influence par la mobilisation de mécanismes démocratiques qui rendent possibles de nouvelles expérimentations et l'adaptation progressive des modes de régulation à l'œuvre.

Tant cet effort de repérage que cette proposition d'agenda de recherche montrent l'étendue et la richesse de la problématique formée par le croisement entre stratégies, espaces et territoires. Ce dossier de La Revue Française de Gestion a permis d'aborder ce sujet, de nombreuses pistes de recherche ont été identifiées, elles pourraient être complétées par une invitation à interroger plus avant l'esthétique des organisations selon leur structuration temporelle et spatiale.

\section{Bibliographie}

Adler P.S. et Heckser C., «Towards Collaborative Communauty» in Heckser C., Adler P.S., The Firm as a Collaborative Communauty, Oxford University Press, 2006.

Aniello V., "The Competitive Mezzogiorno: Some Evidence from The Clothing and Textile Industry», International Journal of Urban and Regional Research, 2001, 25 (2). 
Alvesson M. et Willmott H. (Eds), Studying Management Critically, London, Sage, 2003

Banerjee S. et Linstead S., « Globalization, Multiculturalism and Other Fictions: Colonialism for the New Millennium? », Organization, 2001, Vol. 8, No. 4, 683-722.

Bala V. et Goyal S., «Learning from neighbours», Review of Economic Studies, 1998, 65.

Bell G.G., «Clusters, Networks and Firm Innovativeness», Strategic Management Journal, 2005, 26. p. $287-295$.

Benko G. ; Dunford M. et Heurley J., «Districts industriels: vingt ans de recherche», Espaces et sociétés, $1997, n^{\circ} 88 / 89$ p. 305 - 327.

Blanc C., Pour un écosystème de la croissance, Rapport au Premier Ministre, 2004

Birkinshaw J., «Entrepreneurship in MNC : the characteristics of subsidiary initiatives », Strategic Management Journal, 1997, Vol 18, N³, p.207-229

Boschma R.A., «Editorial: Role of Proximity in Interaction and Performance», Regional Studies, 2005 a ,Vol. 39.1

Boschma R.A., «Proximity and Innovation: A critical Assessment», Regional Studies, 2005 b, Vol. 39.1

Boulba-Olga O., «Les relations entreprises-territoires : un construit institutionnel et social», Economies et sociétés. HS, 2006, n 41. 3/4. p. 477 - 496.

Brown K. ; Burgess J. et Festing M., «Towards a New Conceptualization of Clusters». ANZAM Conference, Sydney, Décembre, 2007.

Callon M., "Le réseau comme forme émergente et comme modalité de coordination» in Réseau et coordination, Callon, Cohendet, Curien, Dalle (eds) Economica, 1999, p. 13 - 64.

Carroué L, Géographie de la mondialisation, Armand Collin, 2002

Cohendet P. ; Llerena P. ; Stahn H. et Umbhauer, The Economics of Networks, Springer Verlag, 1998.

Colletis G. et Rychen F., «Entreprises et Territoires : proximités et développement local» in In Pecqueur B. et Zimmerman J.B. , Economies de proximités, Lavoisier Hermes, 2004

Courpasson D. et Golsorkhi D., "Variations autour d'une lecture dynamique du pouvoir dans les phénomènes stratégiques et organisationnels», Table-Ronde. 17ème conférence AIMS .Nice, 2008

DATAR, La France, puissance industrielle, La Documentation Française, 2004

Davies H. et Ellis P., " Porter's Competitive Advantage of Nations: Time for the Final Judgment ? ", Journal of Management Studies, 37:8, 2000 
Dupuy C. et Torre A., "Confiance et proximité in Economie de proximités»,. In Pecqueur B., Zimmerman J.B. , Economies de proximités, Lavoisier Hermes, 2004

Dyer J.H. et Singh H., «The Relational View: Cooperative Strategy and Sources of Interorganizational Competitive Advantage», Academy of Management Review, 1998, 23 (4) p. 660 - 679.

Elango B., «Geographic Scope of Operations by Multinational Companies: An Exploratory Study of Regional and Global Strategies», European Management Journal, 2004, Vol. 22 n4.

Ehlinger S. ; Perret V. et Chabaud D., «Quelle gouvernance pour les réseaux territorialisés d'organisations ?», Revue Française de Gestion, 2007, n 170. p. 155 - 171.

Fleming P. et Spicer A., «'You Can Chekout Anytime, but You Can Never Leave' : Spatial Boundaries in a High Commitment Organization», Human Relations, 2004, Vol. 57, n¹, 75-94.

Florida R., «Toward the Learning Region», Futures, 1995, Vol. 27. n 5. p. 527 - 536.

Francois H. ; Hirczak M. et Senil N., «Territoire et patrimoine : la co-construction d'une dynamique et de ses ressources», Revue d'Economie Régionale et Urbaine, 2006, n 5. p. 683 - 700.

Frost T., "The geographic sources of foreign subsidiarie's innovations », Strategic Management Journal, 2001, Vol. 22, p.101-123

Gilly J.P. et Wallet T F., «Forms of Proximity, Local Governance and the Dynamics of Local Economic Spaces: The Case of Industrial Conversion Processes», International Journal of Urban and Regional Research, 2001, Vol. 25, n³, p. $553-570$.

Gilly J.P., «Enchevêtrement des espaces de régulation et gouvernance territoriale»; Revue d'Economie Régionale et Urbaine, 2005, $\mathrm{n}^{\circ} 5$.

Gordon I.R. et Mc Cann P., «Industrial Clusters: complexes, agglomeration and/or Social Networks?», Urban Studies, 2000, 37 (3) p. $513-532$.

Gueguen G. ; Pellegrin-Boucher E. et Torres O., «Des stratégies Collectives aux écosystèmes d'affaires : le secteur des logiciels comme illustration». Atelier AIMS stratégies collectives, 2004.

Hall B.H. et Ziezdomis R.H., «The patent paradox revisited: an empirical study of patenting in the US semi-conductor industry», RAND Journal of Economics, 2001, 32, p. 101 - 128.

Hatchuel A., «Quel horizon pour les sciences de gestion ? Vers une théorie de l'action collective» in : Les Nouvelles Fondations des Sciences de Gestion, David, Hatchuel et Laufer (Coord.) Vuibert, 2000, p. 7 - 35. 
Hite J., "Patterns of Multidimensionality among Embedded Network Ties: a Typology of Relational Embeddedness in Emerging Entrepreneurial Firms", Strategic Organization, vol 1, №1, 2003

lansiti M. et Levien R., The Keystone Advantage, Harvard Business School Press, 2004

Karpik L., «Dispositifs de confiance et engagements crédibles», Sociologie du Travail, 1996, n4.

Kirat T. et Lung Y., «Innovations et Proximités : le territoire, lieu de déploiement des processus d'apprentissage» in : Coordination économique et apprentissage des firmes. Lazaric et Monnier (coord.). Economica, 2001

Kristensen P.H. et Zeitlin J., Local Players un Global Games - The Strategic Constitution of a Multinational Corporation, Oxford University Press, 2005

Lauriol J., «Entreprises, Territoires et Développement Durable», In Le développement Durable .Colloque de Cerisy, Heurgon, Landrieu, Eds. Editions de l'Aube. Essai, 2006

Lauriol J., «Stratégies d'entreprises, Développement Durable et Economie de la fonctionnalité : vers des écosystèmes serviciels» in : L'Economie des services pour un Développement Durable. Colloque de Cerisy. L'Harmattan. Prospective Essais et recherche. Heurgon et Landrieu eds p. 257 - 271., 2007 a

Lauriol J., «Functional Economy and Business Development: Towards New Strategy and Institutional Challengers», ANZAM. Conference, Sydney, Décembre., 2007 b

Lavenir F. ; Joubert Bompard A. et Wendling C., L'entreprise et l'hexagone, Les Editions de Bercy, 2001 Le Gales P., «Gouvernance des économies locales en France : à la recherche de la coordination perdue», L'année de la régulation 2004. Presses de Sciences Po, 2004

Loasby B.J., «Industrial Districts as Knowledge Communauty» in : Industry, Space and Competition, Bellet et L'Harmet (coord). E. Pelgar, 2002, p.70 - 85.

Lussault M., L'homme spatial, Seuil, 2007

Maillat D., «Milieux innovateurs et dynamique territoriale» in: Economie industrielle et économie spatiale. Rallet A. Et Torre A. (eds), Economica, 1995

Marshall A., Principles of Economics (9e ed) Mac Millan London, 1961, 1er ed. 1890.

Martin R. et Sunley P., «Deconstructing Clusters: Chaotic Concept of Policy Panacea ?» Journal of Economic Geography, 2003, 3. p 5. 35.

Mcgrath R.G. ; Chen M.J et Macmillan I., "Multimarket Maneuvring in Uncertain Spheres of Influence: Resource Diversion Strategies", Academy of Management Review, 1998, Vol 23, №4, 724-740 
Michalet C.A., « Dynamique des formes de délocalisation et gouvernance des firmes et des Etats », Revue Française de Gestion, N¹77/2007

North D.C., Institutions, Institutional Change and Economic Performance, Cambridge University Press, 1990

O'Mahony S. et Ferraro F., "The Emergence of Governance in an Open Source Communauty», Academy of Management Journal, 2007, Vol. 50 (5). p. 1079 - 1106.

Orléan A., L'analyse économique des conventions, PUF Quadridge, 2004

Pecqueur B. et Zimmermann J.B., Economies de proximités. Hermes, Lavoisier, 2004

Pecqueur B., «Les territoires, créateurs de nouvelles ressources productives : le cas de l'Agglomération Grenobloise», Géographie, économie, société, 2005, nº 7. p. 255 - 268.

Pellenbarg P. et Wever E., International Business Geography - Case studies of corporate firms, Routledge Studies in International Business and the World Economy, 2008

Perrat J. et Zimmermann J.B., «Stratégies des firmes et Dynamiques Territoriales». Notes et études documentaires : les nouveaux enjeux de la proximité, 2003, n 5167, p. 15 - 31.

Perroux F., L'Economie du XXè siècle, Presses Universitaires de France, 1961

Porter M.E., The Competitive Advantage of Nations, London. Mac Millan, 1990

Porter M.E., "Location, clusters and the "New" Microeconomics of Competition», Business Economics, 1998, 33 (1) p. 7 - 17.

Porter M.E., «Location, Competition and Economic Development: local clusters in the Global Economy», Economic Development Quaterly, 2000, 14 (1) p. 15 - 31.

Rallet A., «L'Economie de proximité». Communication à l'Ecole - chercheur INRA . Le Croisic , 1999

Rugman A. et Brewer T, The Oxford Handbook of International Business, Oxford University Press, 2001

Rugman A., The Regional Multinational - MNEs and «Global» Strategic Management, Cambridge University Press, 2005

Schamp E.W. ; Rentmeister B. et Lo U., «Dimensions of Proximity in Knowledge based Networks: The Cases of Investment Banking and Automobile Design», European Planning Studies, 2005, Vol. 12, $n^{\circ} 5$, p. $607-624$. 
Sergot B., « La personnalisation des décisions de localisation : le cas des créations de nouveaux sites dans les secteurs de l'industrie manufacturière et de l'édition de logiciels », Finance Contrôle Stratégie, 2007, Vol 10, $\mathrm{N}^{\circ} 4$

Sierra C., «Proximité(s), interactions technologiques et territoriales : une revue», Revue d'Economie Industrielle, 1997, № 82. p.7 - 38.

Sorenson O. et Baum J.A., "Geography and Strategy Editors' Introduction», Advances in Strategic Management, 2003, Vol. 20. p. 1 - 19

Spicer A. «Beyond the Convergence-Divergence Debate: The Role of Spatial Scales in Transforming Organizational Logic », Organization Studies, 2006, Vol. 27, No. 10, 1467-1483

Storper M., «La géographie des conventions : proximité territoriale, interdépendance hors marché et développement économique» in Economie Industrielle et Economie Spatiale. Rallet et Torre (eds) Economica, 1995, p.111 - 127.

Stuart T.E. et Sorenson O., «The geography of Opportunity: Spatial heterogeneity in : Founding rates and the performance of biotechnology firms», Research Policy, 2003, 32. p. 229-253,

Tannery F. et Laurent P., «Les groupes à la croisée des territoires : géostratégie de l'innovation », Finance Contrôle Stratégie, 2007, Vol 10, N4

The Economist, "The fading lustre of clusters", In A special Report on Innovation, 2007, October $13^{\text {th }}$, 2007

The Economist, « The Challengers - Emerging-market Multinationals », 2008, January 12th

Tyre M. et Von Hippel E., "The situated nature of adaptative learning in organizations", Organization Science, 1997, Vol 8, №1

Torre A. et Rallet A., «Proximity and Localization». Regional Studies, 2005, Vol. 39, 1

Zimmermann J.B., "Grappes d'entreprises et petits mondes : une affaire de proximités», Revue Economique, 2001, Vol. 53, n³ p. 517 - 524.

Westney E., "Geography as a Design Variable », in The Future of the Multinational Company, Bikinshaw J. ; Ghoshal S. ; Markides C. ; Stopford J et Yip G. (eds), Wiley, 2003 